\title{
Mixed-Valent Metals Bridged by a Radical Ligand: Fact, or Fiction Based on Structure-Oxidation State Correlations?
}

Biprajit Sarkar, Srikanta Patra, Jan Fiedler, Raghavan B. Sunoj, Deepa Janardanan, Goutam Kumar Lahiri ${ }^{*}$, Wolfgang Kaim ${ }^{*}$

Supporting Information 
(24) Gaussian03 (Revision C.02) Frisch, M. J.; Trucks, G. W.; Schlegel, H. B.; Scuseria, G. E.; Robb, M. A.; Cheeseman, J. R.; Montgomery, Jr., J. A.; Vreven, T.; Kudin, K. N.; Burant, J. C.; Millam, J. M.; Iyengar, S. S.; Tomasi, J.; Barone, V.; Mennucci, B.; Cossi, M.; Scalmani, G.; Rega, N.; Petersson, G. A.; Nakatsuji, H.; Hada, M.; Ehara, M.; Toyota, K.; Fukuda, R.; Hasegawa, J.; Ishida, M.; Nakajima, T.; Honda, Y.; Kitao, O.; Nakai, H.; Klene, M.; Li, X.; Knox, J. E.; Hratchian, H. P.; Cross, J. B.; Bakken, V.; Adamo, C.; Jaramillo, J.; Gomperts, R.; Stratmann, R. E.; Yazyev, O.; Austin, A. J.; Cammi, R.; Pomelli, C.; Ochterski, J. W.; Ayala, P. Y.; Morokuma, K.; Voth, G. A.; Salvador, P.; Dannenberg, J. J.; Zakrzewski, V. G.; Dapprich, S.; Daniels, A. D.; Strain, M. C.; Farkas, O.; Malick, D. K.; Rabuck, A. D.; Raghavachari, K.; Foresman, J. B.; Ortiz, J. V.; Cui, Q.; Baboul, A. G.; Clifford, S.; Cioslowski, J.; Stefanov, B. B.; Liu, G.; Liashenko, A.; Piskorz, P.; Komaromi, I.; Martin, R. L.; Fox, D. J.; Keith, T.; Al-Laham, M. A.; Peng, C. Y.; Nanayakkara, A.; Challacombe, M.; Gill, P. M. W.; Johnson, B.; Chen, W.; Wong, M. W.; Gonzalez, C.; Pople, J. A., Gaussian, Inc., Wallingford CT, 2004. (b) Gaussian98 (Revision A. 11.4.) Frisch, M. J.; Trucks, G. W.; Schlegel, H. B.; Scuseria, G. E.; Robb, M. A.; Cheeseman, J. R.; Zakrzewski, V. G.; Montgomery, J. A., Jr.; Stratmann, R. E.; Burant, J. C.; Dapprich, S.; Millam, J. M.; Daniels, A.D.; Kudin, K. N.; Strain, M. C.; Farkas, O.; Tomasi, J.; Barone, V.; Cossi, M.; Cammi, R.; Mennucci, B.; Pomelli, C.; Adamo, C.; Clifford, S.; Ochterski, J. W.; Petersson, G. A.; Ayala, P. Y.; Cui, Q.; Morokuma, K.; Malick, D. K.; Rabuck, A. D.; Raghavachari, K.; Foresman, J. B. Cioslowski, J.; Ortiz, J. V.; Stefanov, B. B.; Liu, G.; Liashenko, A.; Piskorz, P.; Komaromi, I.; Gomperts, R.; Martin, R. L.; Fox, D. J.; Keith, T.; Al-Laham, M. A.; Peng, C. Y.; Nanayakkara, A.; Gonzalez, C.; Challacombe, M.; Gill, P. M. W.; Johnson, B.; Chen, W.; Wong, M. W.; Andres, J. L.; Head-Gordon, M.; Replogle, E. S.; Pople, J. A., Gaussian, Inc., Pittsburgh PA, 2002. 
Table S1. Orbital Energies and MO Compositions for $\mathbf{1}$

\begin{tabular}{|c|c|c|c|c|c|}
\hline \multirow{2}{*}{ MO } & \multicolumn{2}{|c|}{ MO energy } & \multicolumn{3}{|c|}{ MO percentage contribution } \\
\hline & a.u. & $\mathrm{cm}^{-1}$ & $\% \mathrm{Ru}$ & \%acac & \%abpy \\
\hline $115(\mathrm{LUMO}+5)$ & -0.00510 & -1129 & 8.25 & 4.41 & 87.34 \\
\hline $114(\mathrm{LUMO}+4)$ & -0.01898 & -4194 & 2.14 & 4.55 & 93.31 \\
\hline 113 (LUMO+3) & -0.02429 & -5323 & 4.76 & 86.97 & 8.27 \\
\hline $112(\mathrm{LUMO}+2)$ & -0.02635 & -5807 & 4.31 & 79.11 & 16.58 \\
\hline $111(\mathrm{LUMO}+1)$ & -0.03158 & -6936 & 2.63 & 18.48 & 78.90 \\
\hline 110 (LUMO) & -0.08716 & -19115 & 22.17 & 4.23 & 73.60 \\
\hline 109 (HOMO) & -0.17477 & -38392 & 61.84 & 30.40 & 7.76 \\
\hline 108 (HOMO-1) & -0.19018 & -41780 & 52.78 & 27.14 & 20.08 \\
\hline 107 (HOMO-2) & -0.19400 & -42586 & 66.31 & 19.72 & 13.97 \\
\hline 106 (HOMO-3) & -0.21990 & -48232 & 11.92 & 82.17 & 5.91 \\
\hline 105 (HOMO-4) & -0.23047 & -50571 & 25.17 & 70.97 & 3.86 \\
\hline 104 (HOMO-5) & -0.24679 & -54200 & 3.11 & 24.02 & 72.86 \\
\hline 103 (HOMO-6) & -0.25164 & -55249 & 2.20 & 60.55 & 37.24 \\
\hline
\end{tabular}


Table S2. Selected List of Vertical Excitations Computed at the TD-DFT/B3LYP//B3LYP/ 6-31G* Level for 1

\begin{tabular}{|c|c|c|c|c|}
\hline $\begin{array}{l}\text { excitation } \\
\text { energy }^{\mathrm{a}} \\
\left(10^{3} \mathrm{~cm}^{-1}\right)\end{array}$ & $\begin{array}{l}\text { oscillator } \\
\text { strength }\end{array}$ & $\varepsilon^{c}$ & $\psi_{\mathrm{o}}-\psi_{\mathrm{v}}^{\mathrm{d}}$ & type of transition \\
\hline $\begin{array}{l}19.395 \\
(515.59)^{b}\end{array}$ & 0.0872 & 6123.8118 & HOMO-1 $\rightarrow$ LUMO $(0.47)^{\mathrm{e}}$ & 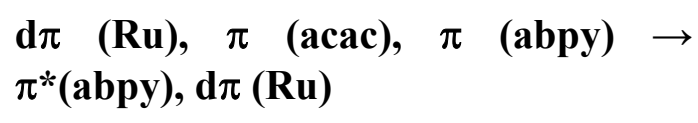 \\
\hline $\begin{array}{l}23.442 \\
(426.59)\end{array}$ & 0.0082 & 575.86304 & HOMO-3 $\rightarrow$ LUMO (0.64) & $\pi(\mathrm{acac}), \mathrm{d} \pi(\mathrm{Ru}) \rightarrow \pi^{*}(\mathrm{abpy}), \mathrm{d} \pi(\mathrm{Ru})$ \\
\hline $\begin{array}{l}24.893 \\
(401.72)\end{array}$ & 0.0174 & 1221.9532 & $\begin{array}{l}\text { HOMO } \rightarrow \text { LUMO+1 }(0.43) \\
\text { HOMO-4 } \rightarrow \text { LUMO }(0.23)\end{array}$ & $\begin{array}{l}\mathrm{d} \pi(\mathrm{Ru}), \pi(\mathrm{acac}) \rightarrow \pi^{*}(\mathrm{abpy}), \pi^{*}(\mathrm{acac}) \\
\pi(\mathrm{acac}), \mathrm{d} \pi(\mathrm{Ru}) \rightarrow \pi^{*}(\mathrm{abpy}), \mathrm{d} \pi(\mathrm{Ru})\end{array}$ \\
\hline $\begin{array}{l}25.358 \\
(394.35)\end{array}$ & 0.0024 & 168.54528 & $\mathrm{HOMO} \rightarrow \mathrm{LUMO}+3(0.59)$ & $\mathrm{d} \pi(\mathrm{Ru}), \pi(\mathrm{acac}) \rightarrow \pi^{*}(\mathrm{acac})$ \\
\hline $\begin{array}{l}25.852 \\
(386.81)\end{array}$ & 0.0032 & 224.72704 & $\begin{array}{l}\mathrm{HOMO} \rightarrow \mathrm{LUMO}+1(0.43) \\
\mathrm{HOMO} \rightarrow \mathrm{LUMO}+2(0.26)\end{array}$ & $\begin{array}{l}\mathrm{d} \pi(\mathrm{Ru}), \pi(\mathrm{acac}) \rightarrow \pi^{*}(\mathrm{abpy}), \pi^{*}(\mathrm{acac}) \\
\mathrm{d} \pi(\mathrm{Ru}), \pi(\mathrm{acac}) \rightarrow \pi^{*}(\mathrm{acac}), \pi^{*}(\mathrm{abpy})\end{array}$ \\
\hline $\begin{array}{l}27.858 \\
(358.97)\end{array}$ & 0.0108 & 758.45376 & $\begin{array}{l}\text { HOMO-5 } \rightarrow \text { LUMO }(0.48) \\
\text { HOMO-6 } \rightarrow \text { LUMO }(0.36)\end{array}$ & $\begin{array}{l}\pi(\text { abpy }), \pi(\text { acac }) \rightarrow \pi^{*}(\text { abpy }), d \pi(R u) \\
\pi(\text { acac }), \pi(\text { abpy }) \rightarrow \pi^{*}(\text { abpy }), d \pi(R u)\end{array}$ \\
\hline $\begin{array}{l}28.818 \\
(347.00)\end{array}$ & 0.0352 & 2471.9974 & HOMO-1 $\rightarrow$ LUMO+1 (0.57) & 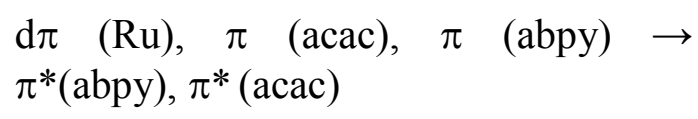 \\
\hline $\begin{array}{l}29.087 \\
(343.80)\end{array}$ & 0.0078 & 547.77216 & $\begin{array}{l}\mathrm{HOMO}-1 \rightarrow \mathrm{LUMO}+3(0.39) \\
\mathrm{HOMO} \rightarrow \mathrm{LUMO}+4(0.30)\end{array}$ & $\begin{array}{l}\mathrm{d} \pi(\mathrm{Ru}), \pi(\mathrm{acac}), \pi(\text { abpy }) \rightarrow \pi^{*}(\mathrm{acac}) \\
\mathrm{d} \pi(\mathrm{Ru}), \pi(\mathrm{acac}) \rightarrow \pi^{*}(\mathrm{abpy})\end{array}$ \\
\hline $\begin{array}{l}29.683 \\
(336.89)\end{array}$ & 0.0148 & 1039.3625 & $\mathrm{HOMO}-2 \rightarrow \mathrm{LUMO}+2(0.47)$ & 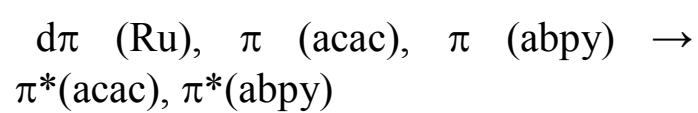 \\
\hline
\end{tabular}

${ }^{a}$ Singlet excitation energies; ${ }^{\mathrm{b}}$ Energy values in nm given in parentheses; ${ }^{\mathrm{c}} \varepsilon$ in $/ \mathrm{M}^{-1} \mathrm{~cm}^{-1} ;{ }^{\mathrm{d}}$ occupied and virtual orbitals; ${ }^{\mathrm{e}}$ transition coefficients. 
Table S3. B3LYP/SDD/6-31G* Optimized Structure Parameters for $(\mathrm{L}) \mathrm{Ru}(\mathrm{acac})_{2}$, L = abpy (1) and abcp

\begin{tabular}{|c|c|c|c|c|c|}
\hline \multicolumn{3}{|c|}{ bond lengths $(\AA)$} & \multicolumn{3}{|c|}{ bond angles $\left({ }^{\circ}\right)$} \\
\hline & $\mathrm{L}=a b p y$ & $\mathrm{~L}=a b c p$ & & $\mathrm{~L}=a b p y$ & $\mathrm{~L}=a b c p$ \\
\hline Ru1-N3 & 2.642 & 1.946 & N3-Ru1-N5 & 77.1 & 76.6 \\
\hline Ru1-N5 & 2.041 & 2.038 & N3-Ru1-O6 & 93.4 & 93.7 \\
\hline Ru1-O6 & 2.053 & 2.047 & N3-Ru1-O8 & 174.4 & 174.0 \\
\hline Rul-O7 & 2.086 & 2.078 & N3-Ru1-O9 & 91.7 & 92.8 \\
\hline Ru1-O8 & 2.096 & 2.092 & N3-Ru1-O7 & 99.4 & 99.5 \\
\hline Ru1-O9 & 2.074 & 2.062 & N5-Ru1-O9 & 94.7 & 93.8 \\
\hline \multirow[t]{9}{*}{ N3-N4 } & 1.300 & 1.305 & N5-Ru1-O8 & 97.3 & 97.4 \\
\hline & & & N5-Ru1-O7 & 176.3 & 176.1 \\
\hline & & & N5-Ru1-O6 & 87.5 & 87.9 \\
\hline & & & O6-Ru1-O7 & 91.5 & 91.6 \\
\hline & & & O6-Ru1-O8 & 85.6 & 84.9 \\
\hline & & & O6-Ru1-O9 & 174.8 & 173.6 \\
\hline & & & O7-Ru1-O8 & 86.2 & 86.4 \\
\hline & & & O7-Ru1-O9 & 86.6 & 87.2 \\
\hline & & & O8-Ru1-O9 & 89.5 & 88.7 \\
\hline
\end{tabular}


Table S4. B3LYP/SDD/6-31G* Optimized Structure Parameters for 2 (Singlet)

\begin{tabular}{|c|c|c|c|}
\hline \multicolumn{2}{|c|}{ bond lengths $(\AA)$} & \multicolumn{2}{|c|}{ bond angles $\left({ }^{\circ}\right)$} \\
\hline Ru1-N1 & 2.022 & N1-Ru1-O3 & 176.4 \\
\hline Ru1-N3 & 2.030 & O1-Ru1-O4 & 176.4 \\
\hline Ru1-O1 & 2.068 & O2-Ru1-N3 & 172.8 \\
\hline $\mathrm{Ru} 1-\mathrm{O} 2$ & 2.083 & N2-Ru2-O6 & 172.8 \\
\hline Ru1-O3 & 2.100 & N4-Ru2-O7 & 176.4 \\
\hline Ru1-O4 & 2.063 & O5-Ru2-O8 & 176.4 \\
\hline $\mathrm{Ru} 2-\mathrm{N} 2$ & 2.030 & N1-Ru1-N3 & 76.8 \\
\hline Ru2-N4 & 2.022 & N1-Ru1-O1 & 96.6 \\
\hline $\mathrm{Ru} 2-\mathrm{O} 5$ & 2.068 & N1-Ru1-O2 & 96.0 \\
\hline Ru2-O6 & 2.083 & N1-Ru1-O4 & 85.6 \\
\hline $\mathrm{Ru} 2-\mathrm{O} 7$ & 2.100 & N3-Ru1-O1 & 89.3 \\
\hline $\mathrm{Ru} 2-\mathrm{O} 8$ & 2.063 & N3-Ru1-O3 & 103.9 \\
\hline N2-N3 & 1.339 & N3-Ru1-O4 & 94.0 \\
\hline C15-N2 & 1.391 & O1-Ru1-O2 & 90.7 \\
\hline \multirow[t]{13}{*}{ C16-N3 } & 1.391 & O1-Ru1-O3 & 87.0 \\
\hline & & O2-Ru1-O3 & 83.3 \\
\hline & & O2-Ru1-O4 & 86.2 \\
\hline & & O3-Ru1-O4 & 90.8 \\
\hline & & N2-Ru2-N4 & 76.8 \\
\hline & & N2-Ru2-O5 & 89.3 \\
\hline & & N2-Ru2-O7 & 103.9 \\
\hline & & N2-Ru2-O8 & 94.0 \\
\hline & & N4-Ru2-O5 & 96.6 \\
\hline & & N4-Ru2-O6 & 96.0 \\
\hline & & N4-Ru2-O8 & 85.6 \\
\hline & & O5-Ru2-O6 & 90.7 \\
\hline & & O5-Ru2-O7 & 87.0 \\
\hline
\end{tabular}




$\begin{array}{ll}\text { O6-Ru2-O7 } & 83.3 \\ \text { O6-Ru2-O8 } & 86.2 \\ \text { O7-Ru2-O8 } & 90.8 \\ \text { tors }(\mathrm{C}-\mathrm{N}=\mathrm{N}-\mathrm{C}) & 0.0\end{array}$


Table S5. B3LYP/SDD/6-31G* Optimized Structure Parameters for 3 (Singlet and Triplet)

\begin{tabular}{|c|c|c|c|c|c|}
\hline \multicolumn{3}{|c|}{ bond lengths $(\AA)$} & \multicolumn{3}{|c|}{ bond angles $\left({ }^{\circ}\right)$} \\
\hline & ${ }^{1} \mathbf{3}$ & ${ }^{3} 3$ & & ${ }^{1} 3$ & ${ }^{3} 3$ \\
\hline Ru1-N1 & 2.027 & 2.055 & N1-Ru1-O3 & 177.4 & 177.4 \\
\hline Ru1-N3 & 2.014 & 2.000 & O1-Ru1-O4 & 176.9 & 177.1 \\
\hline Ru1-O1 & 2.072 & 2.063 & O2-Ru1-N3 & 173.2 & 173.4 \\
\hline Ru1-O2 & 2.085 & 2.079 & N2-Ru2-O7 & 173.2 & 173.4 \\
\hline Ru1-O3 & 2.094 & 2.088 & N4-Ru2-O6 & 177.4 & 177.5 \\
\hline Ru1-O4 & 2.058 & 2.041 & O5-Ru2-O8 & 176.9 & 177.1 \\
\hline Ru2-N2 & 2.014 & 2.000 & N1-Ru1-N3 & 77.0 & 78.0 \\
\hline Ru2-N4 & 2.027 & 2.055 & N1-Ru1-O1 & 95.4 & 94.2 \\
\hline Ru2-O5 & 2.058 & 2.041 & N1-Ru1-O2 & 96.3 & 95.6 \\
\hline Ru2-O6 & 2.094 & 2.088 & N1-Ru1-O4 & 86.3 & 85.9 \\
\hline $\mathrm{Ru} 2-\mathrm{O} 7$ & 2.085 & 2.079 & N3-Ru1-O1 & 91.3 & 89.7 \\
\hline Ru2-O8 & 2.072 & 2.063 & N3-Ru1-O3 & 102.1 & 100.7 \\
\hline N2-N3 & 1.344 & 1.410 & N3-Ru1-O4 & 91.6 & 93.2 \\
\hline $\mathrm{C} 15-\mathrm{N} 2$ & 1.390 & 1.365 & O1-Ru1-O2 & 90.6 & 89.2 \\
\hline \multirow[t]{11}{*}{ C16-N3 } & 1.390 & 1.365 & O1-Ru1-O3 & 91.3 & 87.9 \\
\hline & & & O2-Ru1-O3 & 84.4 & 85.7 \\
\hline & & & O2-Ru1-O4 & 86.6 & 87.9 \\
\hline & & & O3-Ru1-O4 & 91.2 & 92.0 \\
\hline & & & N2-Ru2-N4 & 77.0 & 78.0 \\
\hline & & & N2-Ru2-O5 & 91.6 & 93.3 \\
\hline & & & N2-Ru2-O6 & 102.1 & 100.8 \\
\hline & & & N2-Ru2-O8 & 91.3 & 89.6 \\
\hline & & & N4-Ru2-O5 & 86.3 & 85.9 \\
\hline & & & N4-Ru2-O7 & 96.3 & 95.6 \\
\hline & & & N4-Ru2-O8 & 95.4 & 94.2 \\
\hline
\end{tabular}




$\begin{array}{lll}\text { O5-Ru2-O6 } & 91.2 & 92.1 \\ \text { O5-Ru2-O7 } & 86.6 & 87.9 \\ \text { O6-Ru2-O7 } & 84.4 & 85.7 \\ \text { O6-Ru2-O8 } & 87.1 & 87.9 \\ \text { O7-Ru2-O8 } & 90.6 & 89.3 \\ \text { tors (C-N=N-C) } & 18.3 & 24.8\end{array}$


Table S6. B3LYP/SDD/6-31G* Optimized Structure Parameters for 4

\begin{tabular}{|c|c|c|c|}
\hline \multicolumn{2}{|c|}{ bond lengths $(\AA)$} & \multicolumn{2}{|c|}{ bond angles $\left({ }^{\circ}\right)$} \\
\hline Ru1-N1 & 2.004 & N1-Ru1-O1 & 176.0 \\
\hline Ru1-N3 & 2.030 & O2-Ru1-O4 & 176.6 \\
\hline Ru1-O1 & 2.073 & O3-Ru1-N3 & 172.8 \\
\hline Ru1-O2 & 2.062 & N2-Ru2-O7 & 172.6 \\
\hline Ru1-O3 & 2.074 & N4-Ru2-O6 & 177.3 \\
\hline Ru1-O4 & 2.059 & O5-Ru2-O8 & 176.9 \\
\hline $\mathrm{Ru} 2-\mathrm{N} 2$ & 2.006 & N1-Ru1-N3 & 76.7 \\
\hline Ru2-N4 & 2.020 & N1-Ru1-O2 & 92.4 \\
\hline $\mathrm{Ru} 2-\mathrm{O} 5$ & 2.046 & N1-Ru1-O3 & 96.1 \\
\hline Ru2-O6 & 2.068 & N1-Ru1-O4 & 90.1 \\
\hline $\mathrm{Ru} 2-\mathrm{O} 7$ & 2.082 & N3-Ru1-O2 & 94.6 \\
\hline $\mathrm{Ru} 2-\mathrm{O} 8$ & 2.071 & N3-Ru1-O1 & 102.5 \\
\hline N2-N3 & 1.352 & N3-Ru1-O4 & 88.2 \\
\hline C15-N2 & 1.384 & $\mathrm{O} 1-\mathrm{Ru} 1-\mathrm{O} 2$ & 91.5 \\
\hline \multirow[t]{13}{*}{ C16-N3 } & 1.378 & O1-Ru1-O3 & 84.7 \\
\hline & & O1-Ru1-O4 & 86.1 \\
\hline & & O2-Ru1-O3 & 86.1 \\
\hline & & O3-Ru1-O4 & 91.4 \\
\hline & & N2-Ru2-N4 & 77.0 \\
\hline & & N2-Ru2-O5 & 91.1 \\
\hline & & N2-Ru2-O6 & 102.2 \\
\hline & & N2-Ru2-O8 & 91.4 \\
\hline & & N4-Ru2-O5 & 85.8 \\
\hline & & N4-Ru2-O7 & 95.6 \\
\hline & & N4-Ru2-O8 & 95.7 \\
\hline & & O5-Ru2-O6 & 91.2 \\
\hline & & O5-Ru2-O7 & 87.8 \\
\hline
\end{tabular}




$\begin{array}{ll}\text { O6-Ru2-O7 } & 85.1 \\ \text { O6-Ru2-O8 } & 87.4 \\ \text { O7-Ru2-O8 } & 89.9 \\ \text { tors (C-N=N-C) } & 16.9\end{array}$


Table S7. Orbital Energies for 3 (Triplet)

\begin{tabular}{|c|c|c|c|c|c|}
\hline \multicolumn{3}{|l|}{$\alpha$ set } & \multicolumn{3}{|l|}{$\beta$ set } \\
\hline \multirow{2}{*}{ orbital } & \multicolumn{2}{|l|}{ energy } & \multirow{2}{*}{ orbital } & \multicolumn{2}{|l|}{ energy } \\
\hline & a.u. & $\mathrm{cm}^{-1}$ & & a.u. & $\mathrm{cm}^{-1}$ \\
\hline $174(\mathrm{LUMO}+2)$ & -0.029280 & -06426 & 172 (LUMO+2) & -0.030790 & -06758 \\
\hline $173(\mathrm{LUMO}+1)$ & -0.029960 & -06576 & 171 (LUMO+1) & -0.074820 & -16421 \\
\hline 172 (LUMO) & -0.031830 & -06986 & 170 (LUMO) & -0.097880 & -21482 \\
\hline 171 (HOMO) & -0.145790 & -31997 & 169 (HOMO) & -0.160400 & -35204 \\
\hline 170 (HOMO-1) & -0.181580 & -39853 & 168 (HOMO-1) & -0.178330 & -39139 \\
\hline 169 (HOMO-2) & -0.186940 & -41029 & 167 (HOMO-2) & -0.189320 & -41551 \\
\hline 168 (HOMO-3) & -0.194340 & -42653 & 166 (HOMO-3) & -0.192520 & -42254 \\
\hline 167 (HOMO-4) & -0.199510 & -43788 & 165 (HOMO-4) & -0.197410 & -43327 \\
\hline 166 (HOMO-5) & -0.206910 & -45412 & 164 (HOMO-5) & -0.215300 & -47253 \\
\hline 165 (HOMO-6) & -0.212840 & -46713 & 163 (HOMO-6) & -0.219570 & -48190 \\
\hline 164 (HOMO-7) & -0.223190 & -48985 & 162 (HOMO-7) & -0.221120 & -48531 \\
\hline 163 (HOMO-8) & -0.226660 & -49746 & 161 (HOMO-8) & -0.229810 & -50438 \\
\hline 162 (HOMO-9) & -0.228090 & -50060 & 160(HOMO-9) & -0.231490 & -50807 \\
\hline \multicolumn{3}{|c|}{$\Delta \mathrm{E}_{(\text {LUMO-HOMO) }}=3.10 \mathrm{eV}\left(25011 \mathrm{~cm}^{-1}\right)$} & \multicolumn{3}{|c|}{$\Delta \mathrm{E}_{(\text {LUMO-HOMO) }}=1.70 \mathrm{eV}\left(13722 \mathrm{~cm}^{-1}\right)$} \\
\hline
\end{tabular}


Table S8. Orbital Compositions (\%) of 3 (Triplet) [ $\alpha$ Set $]$

\begin{tabular}{|c|c|c|c|c|}
\hline MO & $\%$ Rul & $\% \mathrm{Ru} 2$ & \%abpy & $\%$ acac \\
\hline $176(\mathrm{LUMO}+4)$ & 2.39 & 2.38 & 93.08 & 2.14 \\
\hline 175 (LUMO+3) & 2.21 & 2.19 & 2.44 & 93.16 \\
\hline $174(\mathrm{LUMO}+2)$ & 1.91 & 1.94 & 1.08 & 95.07 \\
\hline 173 (LUMO+1) & 1.21 & 1.25 & 0.51 & 97.03 \\
\hline 172 (LUMO) & 1.78 & 1.74 & 3.77 & 92.71 \\
\hline 171 (HOMO) & 10.30 & 10.30 & 75.13 & 4.28 \\
\hline 170 (HOMO-1) & 26.45 & 26.58 & 10.28 & 36.69 \\
\hline 169 (HOMO-2) & 27.86 & 27.76 & 4.50 & 39.88 \\
\hline 168 (HOMO-3) & 27.22 & 27.20 & 12.67 & 32.90 \\
\hline 167 (HOMO-4) & 37.65 & 37.59 & 5.30 & 19.45 \\
\hline 166 (HOMO-5) & 31.06 & 31.11 & 9.35 & 28.48 \\
\hline 165 (HOMO-6) & 13.84 & 13.84 & 8.04 & 64.28 \\
\hline 164 (HOMO-7) & 3.88 & 3.87 & 21.08 & 71.17 \\
\hline 163 (HOMO-8) & 6.54 & 6.54 & 41.50 & 45.42 \\
\hline
\end{tabular}


Table S9. Orbital Compositions (\%) of $\mathbf{3}$ (Triplet) [ $\beta$ Set]

\begin{tabular}{|c|c|c|c|c|}
\hline $\mathrm{MO}$ & $\% \mathrm{Ru} 1$ & $\% \mathrm{Ru} 2$ & \%abpy & $\%$ acac \\
\hline $174(\mathrm{LUMO}+4)$ & 2.36 & 2.41 & 1.08 & 94.14 \\
\hline $173(\mathrm{LUMO}+3)$ & 1.51 & 1.66 & 1.21 & 95.62 \\
\hline $172(\mathrm{LUMO}+2)$ & 2.53 & 2.37 & 4.89 & 90.20 \\
\hline 171 (LUMO+1) & 19.95 & 19.98 & 50.50 & 9.57 \\
\hline 170 (LUMO) & 37.48 & 37.45 & 10.70 & 14.38 \\
\hline 169 (HOMO) & 27.01 & 27.05 & 24.71 & 21.23 \\
\hline 168 (HOMO-1) & 34.02 & 34.04 & 7.47 & 24.48 \\
\hline 167 (HOMO-2) & 38.50 & 38.44 & 4.79 & 18.27 \\
\hline 166 (HOMO-3) & 23.00 & 22.96 & 30.23 & 23.81 \\
\hline 165 (HOMO-4) & 37.49 & 37.54 & 5.22 & 19.75 \\
\hline 164 (HOMO-5) & 1.61 & 1.60 & 43.10 & 53.68 \\
\hline 163 (HOMO-6) & 3.14 & 3.11 & 32.67 & 61.08 \\
\hline 162 (HOMO-7) & 4.34 & 4.38 & 5.35 & 85.94 \\
\hline 161 (HOMO-8) & 10.30 & 10.40 & 2.95 & 76.35 \\
\hline
\end{tabular}


Table S10. Selected List of Vertical Excitations Computed at the TD-DFT/B3LYP//B3LYP/ 6-31G*

Level for 3 (Triplet)

\begin{tabular}{|c|c|c|c|c|c|}
\hline $\begin{array}{l}\text { excitation } \\
\text { energy } \\
\left(10^{3} \mathrm{~cm}^{-1}\right)\end{array}$ & $\begin{array}{l}\text { oscillator } \\
\text { strength }\end{array}$ & $\varepsilon^{\mathrm{c}}$ & $\psi_{\mathrm{o}}-\psi_{\mathrm{v}}^{\mathrm{d}}$ & & type of transition \\
\hline $\begin{array}{r}4.075 \\
(2453.59)^{b}\end{array}$ & 0.0124 & 870.817 & HOМO & $\rightarrow \operatorname{LUMO}(0.82)^{\mathrm{e}} \beta$ & $\begin{array}{l}\mathbf{d} \pi\left(\mathrm{Ru}_{1}, \mathrm{Ru}_{2}\right), \pi(\mathrm{abpy}), \pi(\mathrm{acac}) \rightarrow \\
\mathbf{d} \pi\left(\mathrm{Ru}_{1}, \mathbf{R u _ { 2 }}\right), \pi^{*}(\mathrm{acac}), \pi^{*}(\mathrm{abpy})\end{array}$ \\
\hline $\begin{array}{r}12.822 \\
(779.92)\end{array}$ & 0.1511 & 10611.330 & HOMO-3 & $\rightarrow$ LUMO (0.74) $\beta$ & $\begin{array}{l}\mathbf{d} \pi\left(\operatorname{Ru}_{1}, \operatorname{Ru}_{2}\right), \pi(\operatorname{abpy}), \pi(\operatorname{acac}) \rightarrow \\
\mathbf{d} \pi\left(\operatorname{Ru}_{1}, \operatorname{Ru}_{2}\right), \pi^{*}(\operatorname{acac}), \pi^{*}(\operatorname{abpy})\end{array}$ \\
\hline $\begin{array}{r}16.683 \\
(599.41)\end{array}$ & 0.0124 & 870.817 & HOMO-1 & $\rightarrow$ LUMO+1 (0.85) $\beta$ & $\begin{array}{l}\mathbf{d} \pi \quad\left(\mathrm{Ru}_{1}, \mathrm{Ru}_{2}\right), \quad \pi(\mathrm{acac}) \\
\pi^{*}(\mathrm{abpy}), \mathbf{d} \pi\left(\mathrm{Ru}_{1}, \mathrm{Ru}_{2}\right)\end{array}$ \\
\hline $\begin{array}{r}18.952 \\
(527.63)\end{array}$ & 0.0041 & 287.932 & HOMO-3 & $\rightarrow \mathrm{LUMO}+1(0.86) \beta$ & $\begin{array}{l}\mathrm{d} \pi\left(\mathrm{Ru}_{1}, \mathrm{Ru}_{2}\right), \pi(\mathrm{abpy}), \pi(\mathrm{acac}) \rightarrow \\
\pi^{*}(\mathrm{abpy}), \mathrm{d} \pi\left(\mathrm{Ru}_{1}, \mathrm{Ru}_{2}\right)\end{array}$ \\
\hline $\begin{array}{r}18.996 \\
(526.43)\end{array}$ & 0.0059 & 414.340 & HOMO-2 & $\rightarrow$ LUMO $+1(0.85) \beta$ & $\begin{array}{l}\mathrm{d} \pi\left(\mathrm{Ru}_{1}, \mathrm{Ru}_{2}\right), \pi(\mathrm{acac}) \rightarrow \pi^{*}(\mathrm{abpy}), \\
\mathrm{d} \pi\left(\mathrm{Ru}_{1}, \mathrm{Ru}_{2}\right)\end{array}$ \\
\hline $\begin{array}{r}20.090 \\
(497.74)\end{array}$ & 0.0018 & 123.409 & HOMO & $\rightarrow \mathrm{LUMO}+1(0.0) \alpha$ & $\pi(\mathrm{abpy}), \mathrm{d} \pi\left(\mathrm{Ru}_{1}, \mathrm{Ru}_{2}\right) \rightarrow \pi^{*}(\mathrm{acac})$ \\
\hline $\begin{array}{r}20.233 \\
(494.25)\end{array}$ & 0.0032 & 224.727 & HOMO & $\rightarrow \mathrm{LUMO}+2(0.0) \alpha$ & $\pi(\mathrm{abpy}), \mathrm{d} \pi\left(\mathrm{Ru}_{1}, \mathrm{Ru}_{2}\right) \rightarrow \pi^{*}(\mathrm{acac})$ \\
\hline $\begin{array}{r}20.407 \\
(490.04)\end{array}$ & 0.0109 & 765.476 & HOMO & $\rightarrow \mathrm{LUMO}+3(0.0) \alpha$ & $\begin{array}{llll}\pi(\text { abpy }), & \mathbf{d} \pi & \left(\mathbf{R u}_{1}, \mathbf{R u}_{2}\right) & \rightarrow \\
\pi^{*}(\text { acac }) & & \end{array}$ \\
\hline $\begin{array}{r}20.454 \\
(488.89)\end{array}$ & 0.0058 & 407.318 & $\begin{array}{l}\text { HOMO-5 } \\
\text { HOMO }\end{array}$ & $\begin{array}{l}\rightarrow \text { LUMO }(0.65) \beta \\
\rightarrow \text { LUMO+2 }(0.58) \alpha\end{array}$ & $\begin{array}{l}\pi(\text { acac }), \pi(\text { abpy }) \rightarrow \mathrm{d} \pi\left(\mathrm{Ru}_{1}, \mathrm{Ru}_{2}\right), \\
\pi^{*}(\mathrm{acac}), \\
\pi(\mathrm{abpy}), \mathrm{d} \pi\left(\mathrm{Ru}_{1}, \mathrm{Ru}_{2}\right) \rightarrow \pi^{*}(\mathrm{abpy})\end{array}$ \\
\hline
\end{tabular}

${ }^{\mathrm{a}}$ Triplet excitation energies; ${ }^{\mathrm{b}}$ values in nm given in parentheses; ${ }^{\mathrm{c}} \varepsilon$ in $/ \mathrm{M}^{-1} \mathrm{~cm}^{-1} ;{ }^{\mathrm{d}}$ occupied and virtual orbitals; ${ }^{\mathrm{e}}$ transition coefficients 